\title{
Clinical and radiological characteristics of COVID-19 patients without comorbidities
}

\author{
A single-center study
}

\author{
Saffet Ozturk (D) - Esin Kurtulus Ozturk $(\mathbb{D}) \cdot$ Sibel Yildiz Kaya $(\mathbb{D}$
}

Received: 4 January 2021 / Accepted: 20 April 2021 / Published online: 3 June 2021

(C) Springer-Verlag GmbH Austria, part of Springer Nature 2021

\begin{abstract}
Summary
Objective To evaluate the clinical characteristics and detailed imaging features in coronavirus disease 2019 (COVID-19) patients without comorbidities.

Material and methods This retrospective study included laboratory-confirmed and symptomatic COVID-19 patients without comorbid diseases who were admitted to our second level hospital between March 2020 and September 2020. We assessed the clinical, biochemical and imaging diagnostic parameters on admission. The patients were classified as non-severe and progress to severe group and then the initial parameters were compared.

Results We enrolled 135 adult COVID-19 patients, 12 progressed to severe disease during hospitalization. Compared to the non-severe group, patients who progressed to severe were older $(p<0.001)$ and were more likely to manifest coughing $(p=0.011)$ and have higher lactate dehydrogenase (LDH) levels $(p=0.011)$. On chest computed tomography (CT) images, multilobar $(p=0.016)$, peripherally $(p=0.001)$ distributed mixed ground glass opacities and consolidation $(p<0.001)$, crazy paving $(p=0.007)$ and higher total CT severity score $(p<0.001)$ were significantly associated with severe disease.
\end{abstract}

\footnotetext{
S. Ozturk, MD ( $\bowtie)$

Department of Radiology, Sungurlu State Hospital, Çorum 19300 Sungurlu, Turkey

saffetozturk_7@hotmail.com

E. Kurtulus Ozturk, MD

Faculty of Medicine, Department of Radiology, Kutahya University of Health Sciences, 43100 Kütahya, Turkey e.kurtulus@hotmail.com

S. Yildiz Kaya, MD

Department of Infectious Disease and Clinical Microbiology, Sungurlu State Hospital, 19300 Çorum, Turkey y.sibelly@hotmail.com
}

Conclusion Knowledge of the clinical and radiological parameters associated with disease severity might be useful to guide clinical decision-making for COVID-19 patients without comorbidities.

Keywords COVID-19 · Comorbidity · Computed tomography $\cdot$ Severity $\cdot$ Risk factor

\section{Introduction}

In December 2019 pneumonia cases of unknown etiology were reported from Wuhan City, China. The causative agent was found to be a novel coronavirus (2019-nCoV,) and the disease was named as COVID-19 by many coronavirus researchers. Within a short time period similar cases occurred in many countries because of high contagiousness of the virus. The outbreak was first declared a global health emergency and then a pandemic by the World Health Organization [1-3].

Since the coronavirus disease 2019 (COVID-19) emerged as a pandemic, many researchers have been investigating how to fight against this fatal disease, which is causing high mortality for older patients and in patients with comorbidities. Despite the recent use of many vaccines pandemic contagion continues and clinicians are faced with various clinical challenges in diagnostics and management. The clinical course of the disease may differ from patient to patient. It is essential to detect the disease at an early stage so that prevention of the spread and progress of the disease can be undertaken. In order to do this, we need to know more detailed information about this virus. Although most previous studies have mainly focused on the general clinical and imaging findings of patients with COVID-19, there is limited information about the characteristics of patients with no comorbid disease. Thus, this study aimed to evaluate the clinical and ra- 
diological findings of COVID-19 patients without any comorbid diseases and the relationship with disease progression to severe.

\section{Material and methods}

We retrospectively reviewed the clinical and chest computed tomography (CT) findings of laboratoryconfirmed COVID-19 patients from March 2020 to September 2020. All the patients tested positive for COVID-19 by real-time polymerase chain reaction (RT-PCR) from the nasopharyngeal swab and were investigated at admission with a chest CT scan. Inclusion criteria for this study were as follows: COVID-19 infection confirmed by RT-PCR, availability of chest CT scans and being an adult (aged $\geq 18$ years). Cases with any comorbid disease (such as hypertension, diabetes mellitus, chronic obstructive pulmonary disease, cardiovascular disease, cancer, hyperlipidemia, chronic kidney disease and obesity), prior history of previous any type of cancer or immunodeficiency, or not giving consent to the study were excluded. Clinical and imaging data were retrieved from databases of our department. In addition, patients were carefully questioned about comorbid diseases on admission. For eligible cases, demographic characteristics, clinical features, laboratory test results, chest CT findings and outcomes were documented retrospectively.

The CT scans were performed without contrast using a 4-slice CT scanner (ToshibaAquilion; Toshiba Medical Systems Corporation, Otawara, Japan). All scans were obtained using $5 \mathrm{~mm}$ thickness in axial plane with coronal and sagittal reconstructions. All images were analyzed retrospectively in consensus by two experienced radiologists (6 and 8 years experience).

We documented the presence of parenchymal lesions and atypical features, such as mediastinal lymphadenopathy, pleural effusion and pneumothorax on initial CT. All parenchymal lesions were evaluated with respect to morphology (e.g. consolidation, ground-glass opacities, GGO, crazy paving), location (right, left, bilateral lungs, unilobar or multilobar) and distribution (peripheral, perihilar predominant and further as upper zone or lower zone). After review of the findings, the CT scans in each case were classified using the imaging-based classification (Radiological Society of North America chest CT classification system for reporting COVID-19 pneumonia) [4]. Types of parenchymal abnormalities on CT were grouped into four categories: negative for pneumonia, typical, indeterminate and atypical appearances Based on the affected area of the lungs, the total CT severity scores (CT SS) were calculated for each patient using a CT scoring system for COVID-19 patients to quantitatively estimate the pulmonary involvement. Each lung lobe was visually scored as follows: score 0 no involvement; score 1 less than 5\%; score 2, 5-25\%; score 3, 26-49\%; score 4, 50-75\%; and score 5, more than $75 \%$ involvement. The total CT score was calculated by summing the five lobe scores, ranging from 0 (no involvement) to 25 (maximum involvement) [5].

Disease severity was classified into four categories: mild, moderate, severe and critical based on presenting symptoms using guidelines on diagnosis and treatment protocols of pneumonia caused by novel coronavirus [6].

A severe case was defined as a patient who had $\mathrm{SpO}_{2}<94 \%$ on room air at sea level, a ratio of arterial partial pressure of oxygen to fraction of inspired oxygen $\left(\mathrm{PaO}_{2} / \mathrm{FiO}_{2}\right)<300 \mathrm{~mm} \mathrm{Hg}$, respiratory frequency $>30$ breaths $/ \mathrm{min}$, or lung infiltrates $>50 \%$. Also, a critical case was identified as a patient who had respiratory failure, septic shock, and/or multiple organ dysfunction. Then all patients were divided into two groups according to developing progression or not: patients with non-severe disease (mild or moderate) and patients progressed to severe disease (severe or critical) during hospitalization. The role of clinical and imaging parameters on severity of COVID-19 was evaluated by comparison with the two groups.

Data obtained were analyzed using the IBM SPSS Statistics software, version 24.0 (SPSS Inc, Chicago, IL, USA.) For descriptive analyses continuous variables were expressed as median (minimum-maximum) or mean \pm standard deviation and categorical values as frequency and percentage. The Kolmogorov-Smirnov test was used for normality in order to decide for parametric or non-parametric tests. The $\chi^{2}$-test or Fisher's exact test were used to compare the parameters between patients in non-severe group and progressed to severe group. Student's t-test and the Mann-Whitney U-test were used for comparing the means of the independent groups according to normal and nonnormal distribution. A $p$-value $\leq 0.05$ was considered as statistically significant.

This study was approved by our institutional review board (IRB number 275) and written informed consent was obtained from each patient for being included in the study.

\section{Results}

A total of 135 confirmed COVID-19 patients (mean age $39 \pm 13$ years, range $18-64$ years) were included in this study. There were 66 female $(48.9 \%)$ and 69 male (51.1\%) patients and 30 patients had a previous history of contact with a confirmed COVID-19 patient. The most common presenting symptoms were fatigue $(46.7 \%)$, sore throat $(33.3 \%)$, cough $(31.1 \%)$, fever $(26.7 \%)$ and headache $(26.7 \%)$. Diarrhea without fever, as an atypical presentation was observed in 1 patient.

Laboratory results were abnormal in 75 (55.6\%) patients. The most frequent laboratory abnormalities were lymphopenia (33.3\%), elevated C-reactive pro- 
Table 1 Laboratory blood test results

\begin{tabular}{|c|c|c|c|c|}
\hline Laboratory results & All patients $(n=135)$ & Non-severe group $(n=123)$ & Progress-to-severe group $(n=12)$ & $P$ value ${ }^{*}$ \\
\hline Hemoglobin (g/dL) & $13.8 \pm 1.8$ & - & - & - \\
\hline Decreased— $n(\%)$ & $69(53.3)$ & $66(59.1)$ & $3(25)$ & 0.116 \\
\hline White blood cell count $\left(\times 10^{9} / L\right)$ & $5.4 \pm 1.7$ & - & - & - \\
\hline İncreased-n (\%) & $3(2.2)$ & $3(2.4)$ & 0 & 0.230 \\
\hline Decreased- $n(\%)$ & $24(17.8)$ & $21(17.1)$ & $3(25)$ & 0.805 \\
\hline Neutrophil count $\left(\times 10^{9} / L\right)$ & $2.94(1.34-7.71)$ & - & - & - \\
\hline İncreased-n (\%) & $9(6.7)$ & $9(7.3)$ & 0 & 0.232 \\
\hline Decreased- $n(\%)$ & $18(13.3)$ & $15(12.2)$ & $3(25)$ & 0.470 \\
\hline Lymphocyte count ( $\left.\times 10^{9} / L\right)$ & $1.6 \pm 0.6$ & - & - & - \\
\hline Decreased- $n(\%)$ & $45(33.3)$ & $39(31.7)$ & $6(50)$ & 0.403 \\
\hline Monocyte count $\left(\times 10^{9} / \mathrm{L}\right)$ & $0.4(0.11-2.33)$ & - & - & - \\
\hline İncreased-n (\%) & $6(4.4)$ & $6(4.9)$ & 0 & 0.210 \\
\hline Decreased- $n(\%)$ & $30(22.2)$ & $27(22)$ & $3(25)$ & 0.813 \\
\hline Platelet count $\left(\times 10^{9} / \mathrm{L}\right)$ & $198.5 \pm 61.6$ & - & - & - \\
\hline Decreased- $n(\%)$ & $24(17.8)$ & 24 (19.4) & 0 & 0.193 \\
\hline C-reactive protein (mg/L) & $4.40(0.33-34.30)$ & - & - & - \\
\hline İncreased-n (\%) & $66(48.9)$ & $57(46.3)$ & $9(75)$ & 0.058 \\
\hline Alanine aminotransferase (U/L) & $24(10-66)$ & - & - & - \\
\hline İncreased-n (\%) & $24(17.8)$ & $24(19.5)$ & 0 & 0.085 \\
\hline $\begin{array}{l}\text { Aspartate aminotransferase } \\
\text { (U/L) }\end{array}$ & $19(8-141)$ & - & - & - \\
\hline İncreased-n (\%) & $15(11.1)$ & $15(12.2)$ & 0 & 0.228 \\
\hline Bilirubin ( $\mu \mathrm{mol} / \mathrm{L})$ & $0.36(0.16-1.45)$ & - & - & - \\
\hline İncreased-n (\%) & $3(2.2)$ & $3(2.4)$ & 0 & 0.222 \\
\hline Urea $(\mathrm{mmol} / \mathrm{L})$ & $26.6 \pm 9.9$ & - & - & - \\
\hline İncreased-n (\%) & $3(2.2)$ & $3(2.4)$ & 0 & 0.241 \\
\hline Lactate dehydrogenase (U/L) & $217.9 \pm 63.7$ & - & - & - \\
\hline İncreased-n (\%) & $45(33.3)$ & $36(29.3)$ & $9(75)$ & 0.011 \\
\hline
\end{tabular}

tein (CRP) level (48.9\%) and lactate dehydrogenase (LDH) level $(33.3 \%)$ in all patients (Table 1 ).

In total 30 patients were hospitalized and placed under isolation. The others were recommended to undertake home quarantine and were followed as an outpatient. The treatment of the patients was arranged according to the Ministry of Health guidelines. Hydroxychloracine treatment (200 mg twice daily) was used in all 135 patients and azithromycin was added to this treatment in 45 patients. Favipiravir $(600 \mathrm{mg}$ twice daily) was additionally given to 63 patients because of mildly increased symptoms. Low molecular weight heparin prophylaxis (anti-Xa IU $/ 0.8 \mathrm{ml}$, once daily) was performed in 45 patients due to older age.

During the hospitalization $12(8.9 \%)$ patients developed respiratory distress during the clinical course and progressed to severe illness. None of those were deteriorated life threatening condition. The remaining $123(91.1 \%)$ patients were included non-severe patient group. All patients were clinically followed-up after a median time of 15 days (range 1-24 days). For patients who progressed to severe disease, the mean disease progression was $6.2 \pm 2.7$ days. There were no infection-related deaths and all had recovered from COVID-19 and discharged from the hospital at the time of analysis.

Compared to the non-severe group, patients progressed to severe disease were significantly older (59 \pm 5 years, range $56-67$ years, $p<0.001)$ and more likely to suffer from coughing $(p=0.011)$. The LDH levels were found to be significantly higher in the progress to severe group but no significant difference was found in other clinical and laboratory findings between the two groups (Tables 1 and 2).

Pulmonary parenchymal abnormalities were noted on 75 (55.6\%) CT scans whilst 60 cases were negative for pneumonia. According to the CT classification system for reporting COVID-19 pneumonia, typical appearance was detected in 57 cases (Fig. 1), indeterminate appearance in 9 cases (Fig. 2) and atypical appearance in 9 cases (Fig. 3). The most common CT features detected in our study were ground glass opacities (53.3\%) followed by consolidation with ground glass opacities $(33.3 \%)$. The parenchymal lesions showed typical multilobar peripheral involvement with affection of lower zones of the bilateral 
Table 2 Baseline characteristics of the study group

\begin{tabular}{|c|c|c|c|c|}
\hline & All patients & Non-severe group & Progress-to-severe group & $P$ value* \\
\hline Total number of patients-n & 135 & 123 & 12 & - \\
\hline Age years mean \pm standard deviation, (range) & $39 \pm 13(18-67)$ & $37 \pm 12(18-59)$ & $59 \pm 5(56-67)$ & $<0.001$ \\
\hline \multicolumn{5}{|l|}{ Gender } \\
\hline Male- $n(\%)$ & $69(51.1)$ & $60(48.8)$ & $9(75)$ & 0.083 \\
\hline \multicolumn{5}{|l|}{ Signs and symptoms-n (\%) } \\
\hline Fever & $36(26.7)$ & $30(24.4)$ & $6(50)$ & 0.083 \\
\hline Cough & $42(31.1)$ & $33(26.8)$ & $9(75)$ & 0.011 \\
\hline Shortness of breath & $15(11.1)$ & $12(9.8)$ & $3(25)$ & 0.132 \\
\hline Fatigue & $63(46.7)$ & $57(46.3)$ & $6(50)$ & 0.808 \\
\hline Abdominal pain & $12(13.3)$ & $15(12.2)$ & $3(25)$ & 0.201 \\
\hline Sore throat & $45(33.3)$ & $42(34.1)$ & $3(25)$ & 0.750 \\
\hline Headache & $36(26.7)$ & $36(29.3)$ & 0 & 0.065 \\
\hline Diarrhea & $11(8.1)$ & $11(8.9)$ & 0 & 0.332 \\
\hline Loss of smell and taste & $12(8.9)$ & $9(7.3)$ & $3(25)$ & 0.075 \\
\hline
\end{tabular}

Fig. 1 Typical CT imaging features of COVID-19. Consecutive axial chest CT images show multilobar peripherally distributed consolidation with ground glass opacities affecting the bilateral lungs CT SS: 8

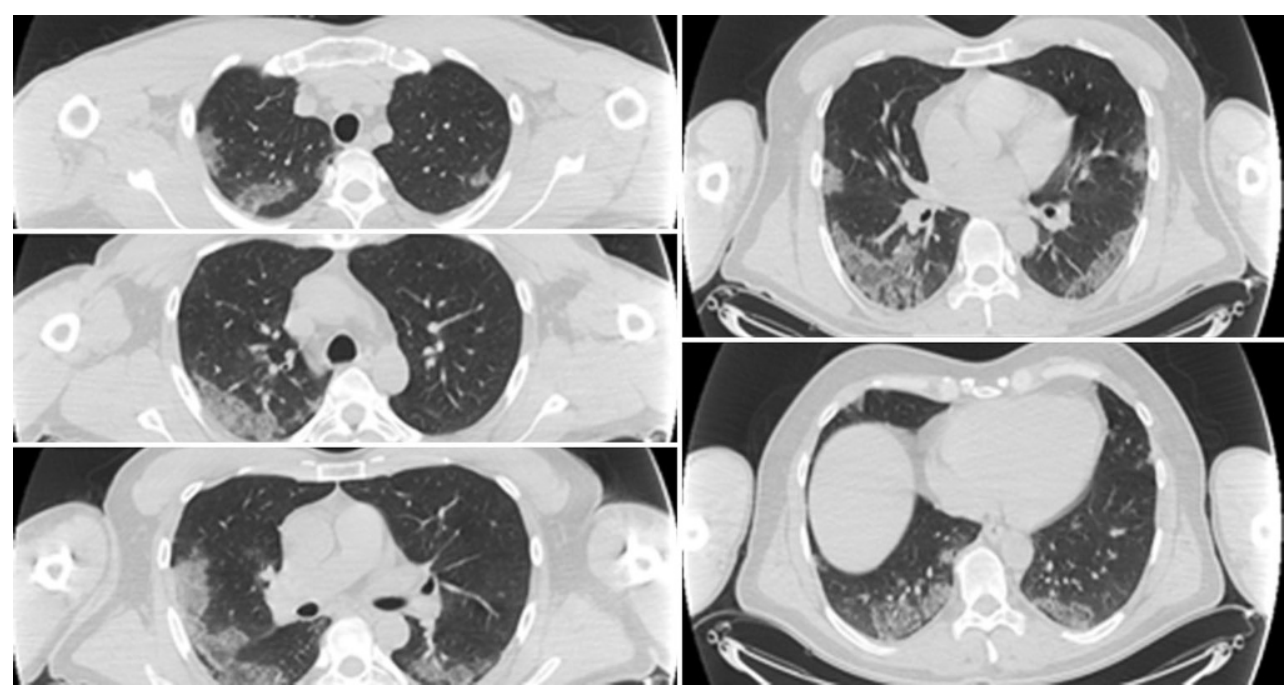

lungs. No pleural effusion, cavitary lesions or pathologic mediastinal lymph nodes were detected in any case. As atypical appearances, isolated lobar consolidation was detected in 1 case, solitary ground glass nodules in 8 patients. The mean CT-SS of all patients was found to be $1.98 \pm 2.2(0-8)$.

Progress to severe patients were more likely to have CT abnormalities including peripheral distribution $(p=0.001)$, multilobar involvement $(p=0.016)$ and GGO with consolidation $(p<0.001)$, Also CT severity score was significantly higher in progressive group than non-severe group $(p<0.001)$. The other chest CT parameters between the two groups were not statistically significant. Initial chest CT findings between non-severe and the progress-to-severe group are summarized in Table 3.

\section{Discussion}

The COVID-19 is a rapidly progressive infectious disease and has affected countries all around the world.
A large number of people have become infected with the coronavirus worldwide and many died from this disease with unknown etiology and treatment $[1,3]$.

COVID-19 exhibits a broad clinical spectrum ranging from mild non-specific upper respiratory symptoms to severe pneumonia, acute respiratory distress syndrome, multiple organ failure and death [7]. Older age ( $\geq 65$ years), living in a nursing home or presence of comorbidities including hypertension, diabetes, morbid obesity, severe heart disease, end-stage kidney disease, moderate to severe asthma, chronic lung disease, chronic liver disease and suppressed immune system (HIV, cancer or steroid treatment ...) have emerged as risk factors for severe disease [8, 9]. Therefore, the majority of the previous studies about COVID-19 were mostly related to these comorbid diseases or non-specific groups. In these studies the most common symptoms were fever, cough, muscle pain or weakness among all patients with or without comorbidities [8]. In our study, unlike the literature, instead of fever, fatigue, sore throat and cough 


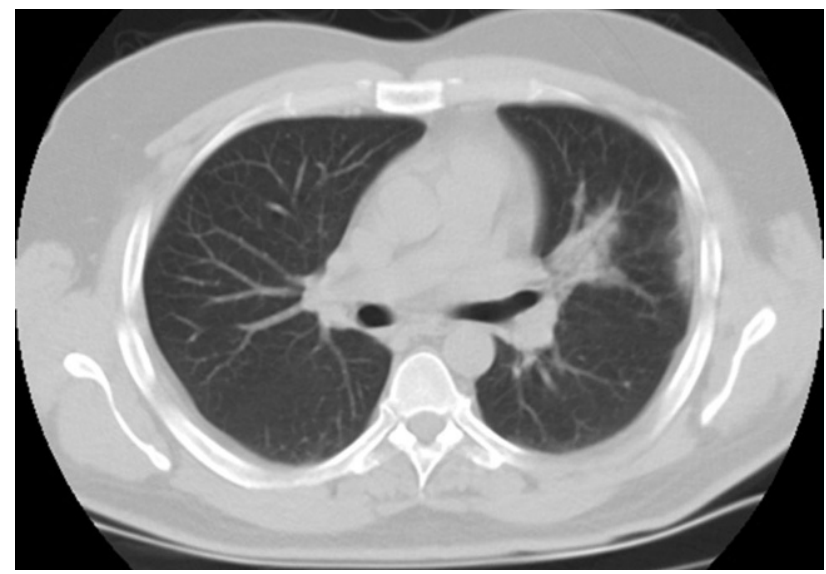

Fig. 2 Indeterminate CT imaging features of COVID-19. Unenhanced axial chest CT image shows unilateral peripheral ground glass opacities with perihilar consolidation CT SS: 2

were the most frequent symptoms. Headache was one of the most common symptoms in our patients. We thought that the absence of additional disease in our population played an important role in the differences in symptomatology.

Li et al. stated that lymphopenia, elevated C-reactive protein and lactate dehydrogenase levels and leukopenia were the most frequent laboratory abnormalities [10]. Our laboratory abnormalities were consistent with previous studies.

To date characteristic chest CT features of COVID19 patients have also been described including multifocal and peripheral distribution of ground glass opacities and consolidation together or pure ground glass opacities, linear reticular opacities, interstitial thickenings and crazy paving pattern [11, 12]. According to our results, typical appearance was observed in almost all of our patients with abnormalities on chest CT scans. Lower zone and peripheral distribution were more affected. No isolated central involvement was detected.

Ground glass opacities were shown to be more frequently observed in the study of Salehi et al. [12]. In our study, similarly ground glass opacities were the most common type of involvement but most of them were detected with consolidation. Of the atypical CT findings, isolated lobar consolidation was detected in 1 patient and a solitary ground glass nodule in 8 patients.

Clinical course and management of COVID-19 depend on the severity of the disease. Patients with no or mild symptoms are able to make a good and speedy recovery at home. Moderate or severe COVID-19 pa-

Table 3 Initial chest CT findings between non-severe and progress-to-severe group

\begin{tabular}{|c|c|c|c|c|}
\hline Parenchymal lesions at baseline chest CT & $\begin{array}{l}\text { All patients } \\
n=135 \\
n(\%)\end{array}$ & $\begin{array}{l}\text { Non-severe } n=123 \\
n(\%)\end{array}$ & $\begin{array}{l}\text { Progress-to-severe } n=12 \\
n(\%)\end{array}$ & $P$ value $^{*}$ \\
\hline Positive for pneumonia & $75(55.6)$ & $63(51.2)$ & $12(100)$ & - \\
\hline \multicolumn{5}{|c|}{ Imaging-based classification system } \\
\hline Negative for pneumonia & $60(44.4)$ & - & - & \multirow[t]{4}{*}{-} \\
\hline Typical appearance & $57(42.2)$ & $48(39)$ & $9(75)$ & \\
\hline Indeterminate appearance & $9(6.7)$ & $6(4.9)$ & $3(25)$ & \\
\hline Atypical appearance & $9(6.7)$ & $9(7.3)$ & 0 & \\
\hline \multicolumn{5}{|l|}{ Morphology of parenchymal lesions } \\
\hline Ground-glass opacities & $72(53.3)$ & $60(48.8)$ & 12 & 0.001 \\
\hline With consolidation (mixed) & $45(33.3)$ & $33(26.8)$ & $12(100)$ & $<0.001$ \\
\hline Pure ground-glass opacities & $27(20)$ & $27(22)$ & 0 & 0.060 \\
\hline Crazy paving & $2(1.5)$ & 0 & $2(16.7)$ & 0.007 \\
\hline İsolated lobar consolidation & $1(0.7)$ & $1(0.8)$ & 0 & 0.911 \\
\hline Solitary ground-glass nodule & $8(5.9)$ & $2(1.6)$ & 0 & 0.830 \\
\hline \multicolumn{5}{|l|}{ Location of parenchymal lesions } \\
\hline Unilobar & $18(13.3)$ & $15(12.2)$ & $3(25)$ & 0.201 \\
\hline Multilobar & 57 (42.2) & $48(39)$ & $9(75)$ & 0.016 \\
\hline Right lung involvement & $15(11.1)$ & $12(9.8)$ & $3(25)$ & 0.084 \\
\hline Left lung involvement & $6(4.4)$ & $3(2.4)$ & $3(25)$ & 0.566 \\
\hline Bilateral involvement & $54(40)$ & $48(39)$ & $6(50)$ & 0.328 \\
\hline \multicolumn{5}{|l|}{ Distribution of parenchymal lesions } \\
\hline Peripheral predominant & $60(44.4)$ & $48(39)$ & $12(100)$ & 0.001 \\
\hline Peripheral and perihilar & $15(11.1)$ & 15 (12.2) & 0 & 0.199 \\
\hline Lower zone predominant & $60(44.4)$ & $54(43.9)$ & $6(50)$ & 0.685 \\
\hline No zonal predominance & 12(8.9) & $9(7.3)$ & $3(25)$ & 0.075 \\
\hline CT severity score & $1.98 \pm 2.2(0-8)$ & $1.68 \pm 1.92(0-6)$ & $5 \pm 2.66(1-8)$ & $<0.001$ \\
\hline
\end{tabular}



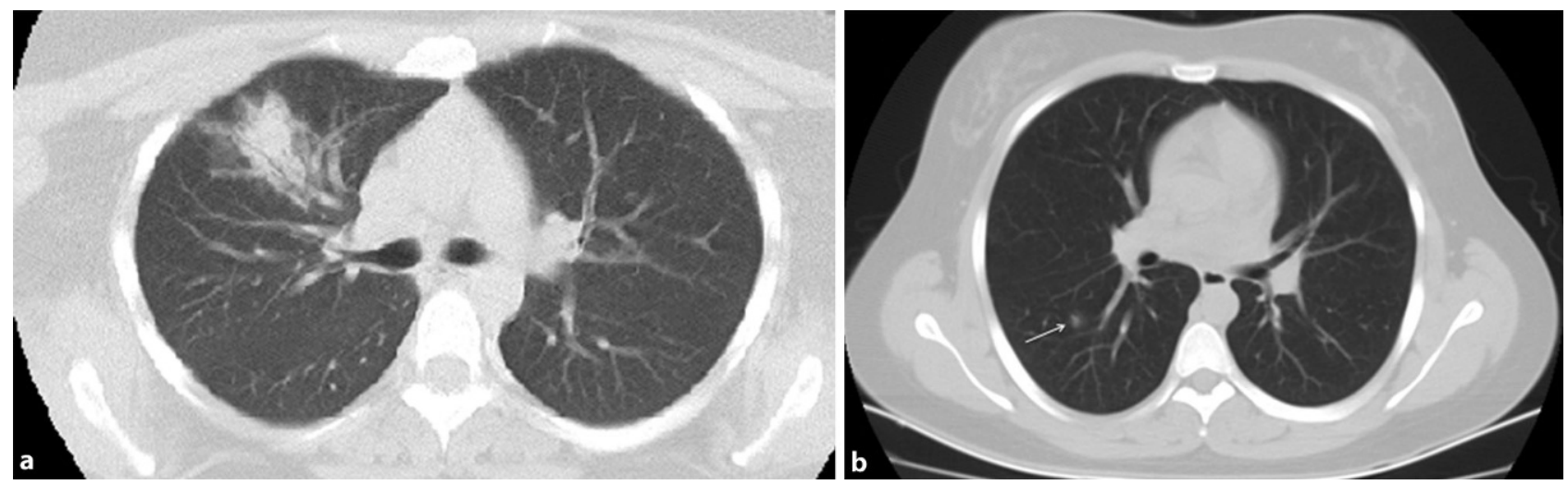

Fig. 3 Atypical CT imaging features for COVID-19. Axial CT images from two different patients showing isolated lobar consolidation (a) (CT SS: 2) and a solitary (arrow) ground glass nodule (b) (CT SS: 1)

tients are often hospitalized for observation, supportive care and treatment [13]. A meta-analysis study noted that patients with underlying chronic illnesses were more likely to contract the virus and their disease has progressed to severe. It was concluded that COVID-19 has an increasingly rapid and severe progression in patients with underlying medical conditions or comorbidities [14]. In the present study, patients without comorbid diseases have a better prognosis and rarely end up with deteriorating outcomes, such as severe pneumonia and respiratory distress when compared with the previous findings reported in the literature.

Song et al. found that disease progression is associated with CT findings [15]. Patients in our study were also more likely to have better clinical and radiological course of COVID-19 when compared with the previous results reported in the literature; however during the follow-up, 12 patients had an increasingly rapid and severe progression of symptoms and CT features even under treatment. We thought that CT findings were getting worse with the clinical course'.

Recently, several studies have investigated the many factors that may affect the clinical course of the infection; however, there has been no consensus whether a parameter or cut-off values should be a prognostic factor for the severity of COVID-19. A comprehensive review reported that the major risk factors of severe clinical course and outcomes of COVID-19 have been identified as old age, male gender, ethnicity, smoking, pregnancy, immunodeficiency, presenting symptoms including fever, dyspnea, gastrointestinal symptoms, comorbid diseases including hypertension, diabetes, obesity, chronic obstructive pulmonary disease, interstitial lung disease, tumor, coagulation disorders, thromboembolism, laboratory abnormalities, such as leukocytosis, lymphopenia, eosinopenia, increased serum levels of Aspartate aminotransferase (AST) and Alanine aminotransferase (ALT), Blood Urea Nitrogen (BUN) and creatine, LDH, D-dimer, troponin I, CRP, procalcitonin, interleukins (6 and $1 \beta$ ) and ferritin. Also, the researchers considered the higher CT pneumonia score and high number of affected pulmonary lobes as significant risk factors [16].

On the other hand, Feng et al. reported that patients in the progressive group were significantly older and more likely to have underlying hypertension. The progressive group had lower lymphocyte counts and albumin, and higher neutrophil-to-lymphocyte ratio, AST, LDH, and CRP levels. On CT images patients in the progressive group had more lobar involvement, with a higher proportion of crazy paving sign and higher CT severity score. Additionally, the researchers stated that older age, higher neutrophil-tolymphocyte ratio, and CT severity score on admission are independent predictors for progression to severe COVID-19 pneumonia [17].

In one study GGO with consolidation was more frequently revealed in progress to severe group whereas pure GGO was more likely to be found in non-severe group [18].

All of the articles mentioned focused on general COVID-19 characteristics not a specific group, whilst we investigated the symptomatic COVID-19 patients with no comorbidities. In the literature, few articles have been published commenting on risk factors for severe COVID-19 patients without comorbidities, unfortunately impact of the detailed imaging findings on disease severity was not assessed in these studies. A multicenter study reported that higher neutrophil to lymphocyte ratio with higher D-dimer levels $(>1 \mu \mathrm{g} / \mathrm{ml})$ on admission might be a potential and reliable predictor for severe progression [19].

Another research noted that LDH and prealbumin levels were relatively reliable markers for the assessment of disease severity in adult patients regardless of the comorbidities [20].

In this current study we found that patients who progressed to severe disease were significantly older $(p<0.001)$ and more likely to present with coughing $(p=0.011)$ and higher LDH levels $(p=0.011)$, but no significant difference was identified in the other clinical characteristics. Additionally, patients in the 
progress-to-severe group were more likely to have multilobar $(p=0.016)$ peripherally $(p=0.001)$ distributed mixed GGO and consolidation $(p<0.001)$, higher proportion of crazy paving sign $(p=0.007)$ and higher total CT severity score $(p<0.001)$.

The current study has some limitations that should be acknowledged. It was a retrospective single-center study with a relatively small sample size. Small sample size particularly of the progressive group limited the statistical power for the comparison between the two group. Nevertheless, we were able to demonstrate significant differences in the initial clinical and imaging findings that might be indicative for disease severity. Larger sample size will further improve the statistical power as well as allow us to compare potential factors with other groups such as patients with comorbidities. Other limitations were wide age range, unavailability of some blood tests, not being able to treat all patients due to second line healthcare institution. Another limitation could be the relatively thick image slice thickness for chest CT that may have influenced image interpretation.

In conclusion, the current study extends our knowledge of COVID-19 patients without comorbidities and contributes to the current understanding and the management of this pandemic. Initial clinical and CT findings may be useful to speculate about disease progression. Old age, coughing, elevated LDH levels, multilobar, peripherally distributed mixed GGO and consolidations, presence of crazy paving sign and higher CT severity score may estimate disease progression in COVID-19 patients without comorbidities. There are still challenges and crucial questions remain to be answered. We believe that there is a need for multicenter studies with larger numbers of patients and wider range of patient groups in order to increase the validity and generalizability of findings.

Funding The authors declared that this study has received no financial support.

Conflict of interest S. Ozturk, E. Kurtulus Ozturk and S. Yildiz Kaya declare that they have no competing interests.

\section{References}

1. WHO. Pneumonia of unknown cause: China. https:// www.who.int/csr/don/05-january-2020-pneumonia-ofunkown-cause-china/en/. Accessed 13 Feb 2020.

2. WHO. Coronavirus disease (COVID-19) technical guidance: early investigations. www.who.int/emergencies/ diseases/novel-coronavirus-2019/technical-guidance/ earlyinvestigations. Accessed 15 Feb 2020.

3. JHWSoE. Coronavirus COVID-19 global cases. gisanddata. maps.arcgis.com/apps/opsdashboard/index.html\#/bda $7594740 f d 40299423467 b 48$ e9ecf6. Accessed 20 Feb 2020.

4. deJaegere TMH, Krdzalic J,Fasen BACM, Kwee RM (2020) Radiological Society of North America Chest CT Classification System for Reporting COVID-19 Pneumonia: Interobserver Variability and Correlation with Reverse-Transcription Polymerase Chain Reaction. Radiology: Cardiothoracic Imaging 2 (3):e200213
5. Pan F, Ye T, Sun P, et al. Time course of lung changes at chest CT during recovery from coronavirus disease 2019 (COVID19). Radiology. 2020;295(3):715-21. https://doi.org/10. 1148 /radiol.2020200370.

6. Wei PF. Diagnosis and Treatment Protocol for Novel Coronavirus Pneumonia (Trial Version 7). Chin Med J (Engl). 2020;133(9):1087-95. https://doi.org/10.1097/ CM9.0000000000000819.

7. Wang D, Hu B, Hu C, et al. Clinical characteristics of 138 hospitalized patients with 2019 novel Coronavirus-infected pneumonia in Wuhan, China. JAMA. 2020; https://doi.org/ 10.1001/jama.2020.1585.

8. Team CC-R. People who are at higher risk for severe illness. https://www.cdc.gov/coronavirus/2019-ncov/ need-extra-precautions/people-at-higher-risk.html. Accessed 14 May 2020.

9. Grasselli G, Zangrillo A, Zanella A, et al. Baseline characteristics and outcomes of 1591 patients infected with SARScoV-2 admitted to ICus of the Lombardy region, Italy. JAMA. 2020; https://doi.org/10.1001/jama.2020.5394.

10. Li LQ, Huang T, Wang YQ, et al. COVID-19 patients' clinical characteristics, discharge rate, and fatality rate of metaanalysis. J Med Virol. 2020;92(6):577-83. https://doi.org/ 10.1002/jmv.25757.

11. Salehi S, Abedi A, Balakrishnan S, Gholamrezanezhad A. Coronavirus disease 2019 (COVID-19): a systematic review of imaging findings in 919 patients. AJR Am J Roentgenol. 2020; https://doi.org/10.2214/AJR.20.23034.

12. Salehi S, Abedi A, Balakrishnan S, Gholamrezanezhad A. Coronavirus disease 2019 (COVID-19) imaging reporting and data system (COVID-RADS) and common lexicon: a proposal based on the imaging data of 37 studies. Eur Radiol. 2020; https://doi.org/10.1007/s00330-020-068630 .

13. Gandhi RT, Lynch JB, Del Rio C. Mild or moderate Covid19. N Engl J Med. 2020; https://doi.org/10.1056/ NEJMcp2009249.

14. Sanyaolu A, Okorie C, Marinkovic A, et al. Comorbidity and its impact on patients with COVID-19. SN Compr Clin Med. 2020; https://doi.org/10.1007/s42399-020-00363-4.

15. SongF, Shi N, Shan F, et al. Emerging 2019 novel coronavirus (2019-ncoV) pneumonia. Radiology. 2020;295(1):210-7. https://doi.org/10.1148/radiol.2020200274.

16. Gao YD, Ding M, Dong X, et al. Risk factors for severe and critically ill COVID-19 patients: A review. Allergy. 2021;76(2):428-55. https://doi.org/10.1111/all.14657.

17. Feng Z, Yu Q, Yao S, et al. Early prediction of disease progression in COVID-19 pneumonia patients with chest CT and clinical characteristics. Nat Commun. 2020;11(1):4968 https://doi.org/10.1038/s41467-020-18786-x.

18. LiY, Shang K, Bian W, et al. Prediction of disease progression in patients with COVID-19 by artificial intelligence assisted lesion quantification. Sci Rep. 2020;10(1):22083. https:// doi.org/10.1038/s41598-020-79097-1.

19. Wang P, Sha J, Meng M, et al. Risk factors for severe COVID19 in middle-aged patients without comorbidities: a multicentre retrospective study. J Transl Med. 2020;18(1):461. https://doi.org/10.1186/s12967-020-02655-8.

20. Chen Z, Zhang F, Hu W, et al. Laboratory markers associated with COVID-19 progression in patients with or without comorbidity: A retrospective study. J Clin Lab Anal. 2021;35(e23644):1. https://doi.org/10.1002/jcla.23644.

Publisher's Note Springer Nature remains neutral with regard to jurisdictional claims in published maps and institutional affiliations. 